\title{
HYPERKÄHLER STRUCTURES WITH TORSION ON NILPOTENT LIE GROUPS
}

\author{
BIRTE FEIX \\ Trinity Hall, Cambridge CB2 1TJ, England \\ e-mail:B.Feix@dpmms.cam.ac.uk \\ and HENRIK PEDERSEN* \\ Dept. of Mathematics and Computer Science, University of Southern Denmark, \\ Campusvej 55, 5230 Odense M, Denmark \\ e-mail:henrik@imada.sdu.dk
}

(Received 16 May, 2002; accepted 20 July, 2002)

\begin{abstract}
Using the classification by Dotti and Fino [3] we show the existence of an HKT metric on a neighbourhood of the centre of any 8-dimensional nilpotent Lie group $G$ with invariant hypercomplex structure. This metric exists globally if the hypercomplex structure is abelian, and in these cases we construct an HKT structure on a neighbourhood of the zero section of the cotangent bundle $T^{*} G$ extending the HKT metric on $G$.
\end{abstract}

2000 Mathematics Subject Classification. 22E25.

1. Introduction. The two-dimensional sigma models studied by physicists force the Riemannian structure of the target space to be compatible with different kinds of quaternionic structures. In the presence of Wess Zumino terms and certain supersymmetries, the target space carries an HKT structure (see, for example, [9]). We begin by recalling some of the facts about these geometries.

A manifold $M$ is hypercomplex if there exists three complex structures $I, J$ and $K$ satisfying the relations of the quaternions $I^{2}=J^{2}=K^{2}=I J K=-1$. A Riemannian manifold $(M, g)$ is called hyperhermitian if it admits a hypercomplex structure such that $g$ is hermitian with respect to $I, J$ and $K$.

An affine connection $\nabla$ on a hyperhermitian manifold $M$ is called hyperkähler with torsion if it satisfies $\nabla g=0, \nabla I=\nabla J=\nabla K=0$ and the torsion tensor $c(X, Y, Z)=g(T(X, Y), Z)$ is totally skew. (Here $T$ is the torsion of $\nabla$.) A manifold is called hyperkähler with torsion (or short HKT) if it is hyperhermitian and possesses a hyperkähler with torsion connection. It is well known (see [7]) that any hypercomplex manifold locally admits a compatible HKT metric.

If there exists a hyperkähler with torsion connection on a hyperhermitian manifold, it is unique; see [7]. Hyperkähler manifolds with torsion are in general not hyperkähler as the Kähler forms corresponding to $I, J$ and $K$ need not be closed. The hyperkähler case corresponds to the case of vanishing torsion and the connection is then the LeviCivita connection.

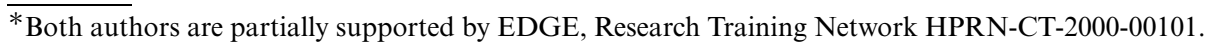


In this paper we shall construct hyperkähler structures with torsion on some nilpotent Lie groups. The first result deals with those 8-dimensional cases where the Lie group admits an invariant hypercomplex structure; in particular, we use the classification by Dotti and Fino [3] of 8-dimensional nilpotent Lie groups with invariant hypercomplex structure. Dotti and Fino [4] have constructed invariant HKT structures on 8-dimensional 2-step nilpotent Lie groups; in particular, they have shown that all invariant HKT structures must have abelian hypercomplex structures. Allowing the metric to be non-invariant, we shall prove the following result.

THeORem A. Let $G$ be any 8-dimensional nilpotent Lie group with invariant hypercomplex structure. Then $G$ admits a compatible HKT structure on a neighbourhood of its centre.

Boyom [2] has described a Lie group structure on $T^{*} G$ using the group structure on $G$ and a flat torsion-free affine connection on $G$. Using this construction when $G$ has an abelian hypercomplex structure we may try to extend the then globally defined HKT metric on $G$ to (a neighbourhood of the zero section of) $T^{*} G$. More precisely, we shall prove the following theorem.

THEOREM B. For any 8-dimensional nilpotent Lie group $G$ with invariant abelian hypercomplex structure there exists an HKT metric on a neighbourhood of the zero section of the cotangent bundle $T^{*} G$ extending the invariant HKT metric on $G$.

This result can be compared to the following theorems on hypercomplex and hyperkähler extensions on (co)tangent bundles.

(i) Let $X$ be a complex manifold equipped with an affine torsion-free connection whose curvature is of type $(1,1)$. Then there exists a hypercomplex structure in a neighbourhood of the zero section of the tangent bundle $T X$ such that the Obata connection restricts to the given connection on the zero section. See [6].

(ii) Let $X$ be any real-analytic Kähler manifold. There exists a hyperkähler metric on a neighbourhood of the zero section of the cotangent bundle $T^{*} X$ that restricts to the given metric on the zero section. See [5].

The result of Theorem B may be regarded as part of the more general extension problem: describe which hermitian manifolds $X$ possess an HKT structure in a neighbourhood of the zero section in $T^{*} X$.

2. The HKT equation. We shall use the following result which allows us to decide whether a hyperhermitian manifold is HKT.

Proposition 1. (See Proposition 2 in [7].) Let $M$ be a hyperhermitian manifold and $F_{1}, F_{2}, F_{3}$ the Kähler forms with respect to $I, J$ and $K$, respectively. Then the metric is $H K T$ if and only if $\partial_{I}\left(F_{2}+i F_{3}\right)=0$, where $\partial_{I}+\bar{\partial}_{I}=d$ is the decomposition of the exterior derivative into types with respect to the complex structure $I$.

Proof. See [7]

The 2-form $F_{2}+i F_{3}$ is of type $(2,0)$. Hence the condition $\partial_{I}\left(F_{2}+i F_{3}\right)=0$ is equivalent to the vanishing of the (3,0)-part of $d\left(F_{2}+i F_{3}\right)$. In the hyperkähler case, since $F_{2}$ and $F_{3}$ are then closed, this condition is satisfied. However, the twistor theory for hyperkähler manifolds uses the fact that $\bar{\partial}_{I}\left(F_{2}+i F_{3}\right)=0$ as the starting point to encode the hyperkähler metric in holomorphic form. Hence the condition of Proposition 1 indicates that the twistor theory for HKT manifolds will have non-holomorphic 
features and will thus be less promising a tool in the construction of HKT structures. For more on the twistor theory see [8]. Since the hypercomplex and hyperkähler extension theorems in [6] and [5] have been proved using twistor theory, their proofs cannot be easily adapted to the case of HKT extensions of hermitian manifolds.

3. Hypercomplex structures on $8 n$-dimensional nilpotent Lie algebras. Let $G$ be an 8n-dimensional 2-step nilpotent Lie group with $\pi_{1} G=0$ and centre $C$ of dimension at least $4 n$. Then we can find invariant 1 -forms $e^{1}, \ldots, e^{8 n}$ on $G$ such that $d e^{k}=0$ for $1 \leq k \leq 4 n$ and $d e^{4 n+j} \in \Lambda^{2}\left\langle e^{1}, \ldots, e^{4 n}\right\rangle$ for $1 \leq j \leq 4 n$. See [10]. Alternatively, $\left[e_{i}, e_{j}\right] \in\left\langle e_{4 n+1}, \ldots e_{8 n}\right\rangle$, for all $i, j \in\{1, \ldots, 4 n\}$, and $\left[e_{4 n+i}, e_{k}\right]=0$, for $i=1, \ldots, 4 n$ and $k=1, \ldots, 8 n$.

We define an invariant almost hypercomplex structure on $G$ by setting $I e^{2 i-1}=e^{2 i}$ for $1 \leq i \leq 4 n$ and $J e^{j}=(-1)^{j+1} e^{2 n+j}$ for $1 \leq j \leq 2 n$ and $4 n+1 \leq j \leq 6 n$ and $K=I J$. In general, the almost complex structures $I, J$ and $K$ may not be integrable.

We now restrict our attention to dimension 8. According to Theorem 2.2 in [3], any 8-dimensional nilpotent Lie group with invariant hypercomplex structure is 2-step nilpotent. It is also clear from the classification in [3] that we can find invariant forms $e^{1}, \ldots, e^{8}$ satisfying the conditions mentioned above such that the complex structures $I, J$ and $K$ are defined as above. Furthermore, in all but one case the centre will be spanned by $e_{5}, \ldots, e_{8}$ (where $e_{1}, \ldots, e_{8}$ are the invariant vector fields dual to $\left.e^{1}, \ldots, e^{8}\right)$. In the exceptional case, the centre is spanned by $e_{4}, e_{5}, \ldots, e_{8}$.

ExAmple 1. The cotangent bundle $T^{*}\left(H_{3} \times \mathbb{R}\right)$ has the algebra $d e^{i}=0$ for $i=1, \ldots, 5$ and $d e^{6}=2 e^{1} \wedge e^{2}, d e^{7}=2 e^{1} \wedge e^{3}$ and $d e^{8}=2 e^{1} \wedge e^{4}$.

The fact that this algebra does indeed describe the cotangent bundle, i. e. is defined on $\mathfrak{g}^{*} \oplus \mathfrak{g}$ where $G=H_{3} \times \mathbb{R}$, can be seen as follows.

Let $X_{1}, X_{2}$ and $X_{3}$ be left-invariant vector fields generating the Lie algebra of the three-dimensional Heisenberg group $H_{3}$ and let $X_{4}$ generate the Lie algebra of $\mathbb{R}$. Then all Lie brackets $\left[X_{i}, X_{j}\right]$ vanish except $\left[X_{1}, X_{2}\right]=X_{3}$. We define a flat torsionfree affine connection $\nabla$ on $G=H_{3} \times \mathbb{R}$ by $\nabla_{X_{1}} X_{2}=X_{3}, \nabla_{X_{1}} X_{1}=-X_{4}$ and all other $\nabla_{X_{i}} X_{j}=0$. Setting $I X_{1}=X_{2}$ and $I X_{3}=X_{4}$ defines a left-invariant complex structure on $G$; the connection $\nabla$ satisfies $\nabla I=0$. Using the process described in Section 5 below, we can use this connection to define a Lie group structure on $T^{*} G$. If we define a basis $X_{1}, \ldots, X_{8}$ of $\mathfrak{g}^{*} \oplus \mathfrak{g}$ by $X_{i}=\left(0, X_{i}\right), X_{4+i}=\left(p_{i}, 0\right), i=1, \ldots, 4$, (where $p_{1} \ldots, p_{4}$ is the dual basis to $\left.X_{1}, \ldots, X_{4}\right)$, the Lie algebra can be described by $\left[X_{1}, X_{2}\right]=X_{3}$, $\left[X_{1}, X_{7}\right]=-X_{6}$ and $\left[X_{1}, X_{8}\right]=X_{5}$. If $p_{1}, \ldots, p_{8}$ is the dual basis to $X_{1}, \ldots, X_{8}$, then letting $e^{1}=2 p_{1}, e^{2}=2 p_{2}, e^{3}=2 p_{8}, e^{4}=2 e_{7}, e^{5}=2 p_{5}, e^{6}=-2 p_{3}, e^{7}=-2 p_{5}$ and $e^{8}=2 p_{6}$ defines the algebra above.

4. Solving the HKT equation in the 8-dimensional case. In this section we shall prove Theorem A. We use the invariant hypercomplex structure on $G$ discussed in Section 3. Our strategy to find an HKT metric on $G$ is to perturb the standard metric $g^{0}$ given in terms of the invariant forms as $g^{0}=\sum_{j=1}^{n}\left(e^{j}\right)^{2}$.

Let $w_{j}=e^{2 j-1}+i e^{2 j}, j=1, \ldots, 4$, be the $(1,0)$ forms with respect to $I$ and define holomorphic functions $z_{j}=x_{2 j-1}+i x_{2 j}, j=1,2$, where $d x_{j}=e^{j}$ for $j=1, \ldots, 4$. We can always find $x_{1}, \ldots, x_{4}$ since $d e^{1}=d e^{2}=d e^{3}=d e^{4}=0$ and $\pi_{1} G=0$. 
If $F_{2}^{0}=g^{0}(\mathrm{~J} \cdot, \cdot)$ and $F_{3}^{0}=g^{0}(K \cdot, \cdot)$, then $F_{2}^{0}+i F_{3}^{0}=w_{1} \wedge w_{2}+w_{3} \wedge w_{4}$, so that

$$
d\left(F_{2}^{0}+i F_{3}^{0}\right)^{3,0}=\left(d w_{3}\right)^{2,0} \wedge w_{4}-w_{3} \wedge\left(d w_{4}\right)^{2,0},
$$

where $\alpha^{p, 0}$ denotes the $(p, 0)$-part with respect to $I$ of the $p$-form $\alpha$. Since $d e^{5}, \ldots, d e^{8} \in \Lambda^{2}\left\langle e_{1}, \ldots e^{4}\right\rangle$, we have $d w_{3}, d w_{4} \in \Lambda^{2}\left\langle w_{1}, \bar{w}_{1}, w_{2}, \bar{w}_{2}\right\rangle$ and thus

$$
\left(d w_{3}\right)^{2,0}=A_{1} w_{1} \wedge w_{2} \quad \text { and } \quad\left(d w_{4}\right)^{2,0}=A_{2} w_{1} \wedge w_{2},
$$

where the coefficients $A_{1}$ and $A_{2}$ are constant, since all forms are $G$-invariant.

We introduce mixed terms into the metric such that $g=g^{0}+\sum_{j=1}^{4} \lambda_{j} g_{j}$ (with real coefficient functions $\left.\lambda_{j}, j=1, \ldots, 4\right)$ remains hyperhermitian. Hence

$$
\begin{aligned}
& g_{1}=e^{1} e^{5}+e^{2} e^{6}+e^{3} e^{7}+e^{4} e^{8}, \\
& g_{2}=e^{1} e^{6}-e^{2} e^{5}-e^{3} e^{8}+e^{4} e^{7}, \\
& g_{3}=e^{1} e^{7}+e^{2} e^{8}-e^{3} e^{5}-e^{4} e^{6}, \\
& g_{4}=e^{1} e^{8}-e^{2} e^{7}+e^{3} e^{6}-e^{4} e^{5} .
\end{aligned}
$$

Let $\alpha_{j}=g_{j}(J \cdot, \cdot)$ and $\beta_{j}=g_{j}(K \cdot, \cdot), j=1, \ldots, 4$. Then

$$
\begin{aligned}
& \alpha_{1}+i \beta_{1}=w_{1} \wedge w_{4}-w_{2} \wedge w_{3}, \\
& \alpha_{2}+i \beta_{2}=i w_{1} \wedge w_{4}+i w_{2} \wedge w_{3}, \\
& \alpha_{3}+i \beta_{3}=-w_{1} \wedge w_{3}-w_{2} \wedge w_{4}, \\
& \alpha_{4}+i \beta_{4}=-i w_{1} \wedge w_{3}+i w_{2} \wedge w_{4} .
\end{aligned}
$$

Choose $\lambda_{1}-i \lambda_{2}=-A_{2} z_{1}$ and $\lambda_{3}-i \lambda_{4}=A_{1} z_{1}$. We have

$$
\begin{aligned}
d\left(F_{2}+i F_{3}\right)^{3,0}= & d\left(F_{2}^{0}+i F_{3}^{0}\right)^{3,0}+\sum_{j=1}^{4} d\left(\lambda_{j}\left(\alpha_{j}+i \beta_{j}\right)\right)^{3,0} \\
= & A_{1} w_{1} \wedge w_{2} \wedge w_{4}-A_{2} w_{1} \wedge w_{2} \wedge w_{3}-d\left(-A_{2} z_{1}\right)^{1,0} \wedge w_{2} \wedge w_{3} \\
& -d\left(A_{1} z_{1}\right)^{1,0} \wedge w_{2} \wedge w_{4}=0,
\end{aligned}
$$

since $d\left(\alpha_{j}+i \beta_{j}\right)^{3,0}=0$ and $d\left(\lambda_{1}+i \lambda_{2}\right)^{1,0}=d\left(\lambda_{3}+i \lambda_{4}\right)^{1,0}=0$. Hence $g$ satisfies the HKT equation.

We recall from the previous section that the centre $C$ corresponds to $x_{1}=x_{2}=x_{3}=x_{4}=0$ or $x_{1}=x_{2}=x_{3}=0$ in the exceptional case respectively. Hence $\left.g\right|_{C}=\left.g^{0}\right|_{C}$, but $g^{0}$ is clearly positive definite and thus $g$ is positive definite in a neighbourhood of $C$ in $G$ concluding the proof of Theorem A.

REMARK 1. If the hypercomplex structure is abelian, i. e. for any $(1,0)$-form $\alpha$ the exterior derivative $d \alpha$ is of type $(1,1)$, the constants $A_{1}$ and $A_{2}$ vanish. Hence the standard metric $g^{0}$ is HKT and thus there exists a global HKT metric on $G$. This is also true in dimension $8 n$ for arbitrary $n$.

EXAMPLE 2. We continue Example 1. For the cotangent bundle $T^{*}\left(H_{3} \times \mathbb{R}\right)$ we have $A_{1}=0$ and $A_{2}=1$, because $d w_{3}=d\left(e^{5}+i e^{6}\right)=\frac{i}{2}\left(w_{1}+\bar{w}_{1}\right) \wedge\left(\bar{w}_{1}-w_{1}\right)=i w_{1} \wedge \bar{w}_{1}$ and $d w_{4}=d\left(e^{7}+i e^{8}\right)=\left(w_{1}+\wedge \bar{w}_{1}\right) \wedge w_{2}$.

The case of $T^{*}\left(H_{3} \times \mathbb{R}\right)$ can also be regarded as an example of an HKT extension to the cotangent bundle of the hermitian metric $g=p_{1}^{2}+p_{2}^{2}+p_{3}^{2}+p_{4}^{2}$ on $H_{3} \times \mathbb{R}$. To 
adopt this point of view we have to choose the solution $\lambda_{1}+i \lambda_{2}=0$ and $\lambda_{3}+i \lambda_{4}=z_{2}$ of the HKT equation. The metric $g$ will then be positive definite in a neighbourhood of the zero section of $T^{*}\left(H_{3} \times \mathbb{R}\right)$.

5. Lifting the algebra to the cotangent bundle. If $G$ is a Lie group possessing a flat torsion-free affine connection $\nabla$ we can define a Lie algebra structure on $\mathfrak{g}^{*} \oplus \mathfrak{g}$ (and thus a Lie group structure on $T^{*} G$ ) as follows; (cf. [2]). If $(\alpha, X),(\beta, Y) \in \mathfrak{g}^{*} \oplus \mathfrak{g}$, then we define

$$
[(\alpha, X),(\beta, Y)]=\left(\alpha \circ \nabla_{Y}-\beta \circ \nabla_{X},[X, Y]\right)
$$

LemMa 1. (Barberis [1].) If $G$ is 2-step nilpotent and carries an invariant hypercomplex structure which preserves the centre, then the Obata connection $\nabla$ on $G$ is flat.

Proof. The Obata connection can be expressed in terms of Lie brackets and the complex structures $I, J$ and $K$ (see, for example, [1]) as

$$
\begin{aligned}
\nabla_{X} Y= & \frac{1}{2}[X, Y]+\frac{1}{12}(I([J X, K Y]+[J Y, K X])+J([K X, I Y]+[K Y, I X]) \\
& +K([I X, J Y]+[I Y, J X]))+\frac{1}{6}(I[I X, Y]+[I Y, X]) \\
& +J([J X, Y]+[J Y, X])+K([K X, Y]+[K Y, X])),
\end{aligned}
$$

but then $\nabla_{X} \nabla_{Y} Z=0$ and $\nabla_{[X, Y]} Z=0$. Hence the Obata connection is flat.

We can therefore use the Obata connection (which is always torsion-free) to define a Lie group structure on $T^{*} G$, where $G$ is any nilpotent 8-dimensional Lie group with invariant hypercomplex structure preserving the centre. From the classification [3] we know that we only have to exclude one exceptional group and in all other cases the centre will be 4-dimensional. The invariant hypercomplex structure on $G$ lifts to an integrable hypercomplex structure on $T^{*} G$.

We now describe the resulting algebra structure on $\mathfrak{g}^{*} \oplus \mathfrak{g}$ in more detail. Let $e_{1}, \ldots, e_{8}$ be a basis of invariant vector fields on $G$ such that the centre is $\left\langle e_{5}, \ldots, e_{8}\right\rangle$. If $e^{1}, \ldots, e^{8}$ are the dual forms then $d e^{1}=d e^{2}=d e^{3}=d e^{4}=0$ and $d e^{5}, d e^{6}, d e^{7}, d e^{8} \in \Lambda^{2}$ $\left\langle e^{1}, \ldots, e^{4}\right\rangle$. For $j=1, \ldots, 4$ define

$$
f_{j}=\left(e^{4+j}, 0\right), \quad f_{4+j}=\left(0, e_{j}\right), \quad f_{8+j}=\left(e^{j}, 0\right), \quad f_{12+j}=\left(0, e_{4+j}\right) .
$$

Then $f_{1}, \ldots, f_{16}$ define a basis of invariant vector fields on $T^{*} G$.

For any $(\alpha, X) \in \mathfrak{g}^{*} \oplus \mathfrak{g}$ and $1 \leq j \leq 4$

$$
\left[(\alpha, X),\left(e^{j}, 0\right)\right]=\left(-e^{j} \circ \nabla_{X}, 0\right)=0,
$$

since $\nabla_{X} Y \in\left\langle e_{5}, \ldots e_{8}\right\rangle$ for any $Y \in \mathfrak{g}$ using the formula (1) for the Obata connection in terms of Lie brackets and the fact that any Lie bracket lies in the centre of $\mathfrak{g}$ which is preserved by the hypercomplex structure. Hence $f_{9}, \ldots, f_{12}$ are in the centre of $\mathfrak{g}^{*} \oplus \mathfrak{g}$. Also

$$
\left[(\alpha, X),\left(0, e_{4+j}\right)\right]=\left(\alpha \circ \nabla_{e_{4+j}}, 0\right)=0
$$


since $\left[X, e_{4+j}\right]=0$ as $e_{4+j}$ is in the centre of $\mathfrak{g}$ and thus also $\nabla_{e_{4+j}}=0$ using formula (1) for the Obata connection in terms of Lie brackets; hence $f_{13}, \ldots f_{16}$ are also in the centre.

The Lie group $T^{*} G$ is 2-step nilpotent because any Lie bracket $\left[f_{p}, f_{q}\right]$ lies in the centre. This is obvious if $p \geq 9$ or $q \geq 9$ (as the bracket then vanishes) or if $5 \leq p, q \leq 8$ (since $\left[e_{p-4}, e_{q-4}\right]$ is in the centre of $\mathfrak{g}$ ). If $1 \leq p, q \leq 4$, then $\left[f_{p}, f_{q}\right]=\left[\left(e^{4+p}, 0\right),\left(e^{4+q}, 0\right)\right]=0$. If $1 \leq p \leq 4$ and $5 \leq q \leq 8$, then

$$
\left[f_{p}, f_{q}\right]=\left(e^{4+p} \circ \nabla_{e_{q-4}}, 0\right) \in\left\langle\left(e^{1}, 0\right), \ldots,\left(e^{4}, 0\right)\right\rangle=\left\langle f_{9}, \ldots, f_{12}\right\rangle,
$$

since $\nabla_{e_{p}} e_{l}=0$ for $l=5, \ldots, 8$. Thus, for any $1 \leq l \leq 4$,

$$
d f^{8+l} \in\left\langle f^{j} \wedge f^{4+k} ; 1 \leq j, k \leq 4\right\rangle \quad \text { and } \quad d f^{12+l} \in\left\langle f^{4+j} \wedge f^{4+k} ; 1 \leq j, k \leq 4\right\rangle
$$

and also $d f^{j}=0$, for $j=1, \ldots, 8$.

Note that the basis of invariant 1 -forms $f^{1}, \ldots, f^{16}$ is actually of the type described in Section 3.

6. An HKT metric on the cotangent bundle. If the invariant hypercomplex structure on an 8-dimensional nilpotent Lie group $G$ is abelian, the standard metric $g^{0}$ is a global HKT metric on $G$ and we will extend it to a neighbourhood of the zero section of the cotangent bundle and thus prove Theorem B.

Examining the classification of such Lie groups by Dotti-Fino [3], we notice that there are only three examples with abelian hypercomplex structure, namely the following trivial extensions of Heisenberg groups: $H_{5}^{\mathbb{R}} \times \mathbb{R}^{3}, H_{3}^{\mathbb{C}} \times \mathbb{C}$ and $H_{1}^{\mathbb{A}} \times \mathbb{R}$.

All three cases will be solved using the following ansatz for a hyperhermitian metric on $T^{*} G$ :

$$
g=g^{0}+\sum_{l=0}^{8} \mu_{l} h_{l},
$$

where $g^{0}=\sum_{j=1}^{16}\left(f^{j}\right)^{2}, h_{0}=\sum_{j=1}^{4}\left(f^{j}\right)^{2}$ and for $j=1, \ldots, 4$,

$$
\begin{aligned}
h_{j} & =f^{1} f^{4+j}+f^{2} I f^{4+j}+f^{3} J f^{4+j}+f^{4} K f^{4+j}, \\
h_{4+j} & =f^{5} f^{8+j}+f^{6} I f^{8+j}+f^{7} J f^{8+j}+f^{8} K f^{8+j} .
\end{aligned}
$$

Let the corresponding Kähler forms be $\alpha_{l}=h_{l}(J \cdot, \cdot)$ and $\beta_{l}=h_{l}(K \cdot, \cdot), l=0, \ldots, 8$, and let $v_{p}=f^{2 p-1}+i f^{2 p}, p=1, \ldots, 8$, be a basis of $(1,0)$-forms with respect to $I$ on $T^{*} G$. Then

$$
\begin{aligned}
& \alpha_{0}+i \beta_{0}=v_{1} \wedge v_{2}+v_{3} \wedge v_{4}, \\
& \alpha_{1}+i \beta_{1}=v_{1} \wedge v_{4}-v_{2} \wedge v_{3}, \\
& \alpha_{2}+i \beta_{2}=i v_{1} \wedge v_{4}+i v_{2} \wedge v_{3}, \\
& \alpha_{3}+i \beta_{3}=-v_{1} \wedge v_{3}-v_{2} \wedge v_{4}, \\
& \alpha_{4}+i \beta_{4}=-i v_{1} \wedge v_{3}+i v_{2} \wedge v_{4}, \\
& \alpha_{5}+i \beta_{5}=v_{3} \wedge v_{6}-v_{4} \wedge v_{5}, \\
& \alpha_{6}+i \beta_{6}=i v_{3} \wedge v_{6}+i v_{4} \wedge v_{5}, \\
& \alpha_{7}+i \beta_{7}=-v_{3} \wedge v_{5}-v_{4} \wedge v_{6}, \\
& \alpha_{8}+i \beta_{8}=-i v_{3} \wedge v_{5}+i v_{4} \wedge v_{6} .
\end{aligned}
$$


In order to produce an HKT metric we need to choose $\mu_{0}, \ldots, \mu_{8}$ such that

$$
d\left(F_{2}+i F_{3}\right)^{3,0}=d\left(F_{2}^{0}+i F_{3}^{0}\right)^{3,0}+\sum_{j=0}^{8} d\left(\mu_{j}\left(\alpha_{j}+i \beta_{j}\right)^{3,0}=0\right.
$$

where $F_{2}^{0}+i F_{3}^{0}=g^{0}(J \cdot, \cdot)+i g^{0}(K \cdot, \cdot)$.

We introduce holomorphic functions $q_{1}, \ldots, q_{4}$ by requiring $d q_{j}=v_{j}$, for $j=1, \ldots, 4$. This choice is possible since $d v_{1}=\cdots=d v_{4}=0$ and $\pi_{1}\left(T^{*} G\right)=\pi_{1} G=0$.

Case 1. $H_{5}^{\mathbb{R}} \times \mathbb{R}^{3}$. The algebra on $H_{5}^{\mathbb{R}} \times \mathbb{R}^{3}$ is given by

$$
\begin{aligned}
& d e^{i}=0, \quad \text { for } i=1, \ldots 7 \\
& d e^{8}=e^{1} \wedge e^{4}-e^{2} \wedge e^{3}
\end{aligned}
$$

Let $e_{1}, \ldots, e_{8}$ be the dual basis. Using formula 1 we compute the Obata connection as

$$
\nabla_{e_{1}} e_{1}=-\nabla_{e_{1}} e_{2}=-\nabla_{e_{3}} e_{3}=-\frac{1}{2} e_{5}
$$

All other $\nabla_{e_{i}} e_{j}$ can be obtained from these using $\nabla I=\nabla J=\nabla K=0$. Using the notation from Section 5 we get

$$
\begin{aligned}
d f^{i} & =0, \text { for } i=1, \ldots, 8,13,14,15, \\
d f^{9} & =\frac{1}{2}\left(f^{1} \wedge f^{5}+f^{2} \wedge f^{6}+f^{3} \wedge f^{7}-f^{4} \wedge f^{8}\right), \\
d f^{10} & =\frac{1}{2}\left(-f^{1} \wedge f^{6}+f^{2} \wedge f^{5}+f^{3} \wedge f^{8}+f^{4} \wedge f^{7}\right), \\
d f^{11} & =\frac{1}{2}\left(-f^{1} \wedge f^{7}-f^{2} \wedge f^{8}+f^{3} \wedge f^{5}-f^{4} \wedge f^{6}\right), \\
d f^{12} & =\frac{1}{2}\left(f^{1} \wedge f^{8}-f^{2} \wedge f^{7}+f^{3} \wedge f^{6}+f^{4} \wedge f^{5}\right), \\
d f^{16} & =f^{5} \wedge f^{8}-f^{6} \wedge f^{7},
\end{aligned}
$$

and therefore

$$
\begin{gathered}
d v_{i}=0 \text { for } i=1, \ldots, 4 \quad \text { and } \quad\left(d v_{7}\right)^{2,0}=\left(d v_{8}\right)^{2,0}=0 \\
\left(d v_{5}\right)^{2,0}=\frac{1}{2} v_{2} \wedge v_{4} \quad \text { and } \quad\left(d v_{6}\right)^{2,0}=\frac{1}{2} v_{2} \wedge v_{3} .
\end{gathered}
$$

Choose $\mu_{0}=0$ and

$$
\begin{aligned}
\mu_{1}+i \mu_{2} & =\frac{1}{4}\left(-q_{2} \bar{q}_{4}+\bar{q}_{2} \bar{q}_{4}+\bar{q}_{1} q_{3}\right), \\
\mu_{3}+i \mu_{4} & =\frac{1}{4}\left(q_{2} \bar{q}_{3}-\bar{q}_{2} \bar{q}_{3}-\bar{q}_{1} q_{4}\right), \\
\mu_{5}+i \mu_{6} & =0 \\
\mu_{7}+i \mu_{8} & =\frac{1}{2}\left(-q_{2}+\bar{q}_{2}\right) .
\end{aligned}
$$


Then the ansatz (2) satisfies the HKT equation for $T^{*}\left(H_{5}^{\mathbb{R}} \times \mathbb{R}^{3}\right)$.

Case 2. $H_{3}^{\mathbb{C}} \times \mathbb{C}$. The algebra on $H_{3}^{\mathbb{C}} \times \mathbb{C}$ is given by

$$
\begin{aligned}
d e^{i} & =0, \quad \text { for } i=1, \ldots 6 \\
d e^{7} & =e^{1} \wedge e^{3}+e^{2} \wedge e^{4} \\
d e^{8} & =e^{1} \wedge e^{4}-e^{2} \wedge e^{3}
\end{aligned}
$$

Note that we use the abelian hypercomplex structure here. There is also a nonabelian complex structure on $H_{3}^{\mathbb{C}}$ by regarding this group as the complexification of $H_{3}^{\mathbb{R}}$.

The Obata connection is given by

$$
\nabla_{e_{1}} e_{1}=-\nabla_{e_{2}} e_{2}=-e_{5} \text { and } \nabla_{e_{3}} e_{3}=\nabla_{e_{4}} e_{4}=0 .
$$

We obtain

$$
\begin{aligned}
d f^{i} & =0, \quad \text { for } i=1, \ldots, 8,13,14, \\
d f^{9} & =f^{1} \wedge f^{5}+f^{2} \wedge f^{6}, \\
d f^{10} & =-f^{1} \wedge f^{6}+f^{2} \wedge f^{5} \\
d f^{11} & =f^{3} \wedge f^{5}-f^{4} \wedge f^{6}, \\
d f^{12} & =f^{3} \wedge f^{6}+f^{4} \wedge f^{5} \\
d f^{15} & =f^{5} \wedge f^{7}+f^{6} \wedge f^{8} \\
d f^{16} & =f^{5} \wedge f^{8}-f^{6} \wedge f^{7} .
\end{aligned}
$$

Therefore $d v_{i}=0$, for $i=1, \ldots, 4$, and $\left(d v_{7}\right)^{2,0}=\left(d v_{8}\right)^{2,0}=0$. Also

$$
\left(d v_{5}\right)^{2,0}=0 \text { and }\left(d v_{6}\right)^{2,0}=v_{2} \wedge v_{3} .
$$

Choose $\mu_{0}=-\bar{q}_{3} q_{3}$ and

$$
\begin{aligned}
\mu_{1}+i \mu_{2} & =-\frac{1}{2}\left(q_{2} \bar{q}_{4}+\bar{q}_{1} q_{3}\right), \\
\mu_{3}+i \mu_{4} & =\frac{1}{2}\left(q_{2} \bar{q}_{3}+\bar{q}_{1} q_{4}\right), \\
\mu_{5}+i \mu_{6} & =0 \\
\mu_{7}+i \mu_{8} & =-q_{2} .
\end{aligned}
$$

Then the ansatz (2) satisfies the HKT equation for $T^{*}\left(H_{3}^{\mathbb{C}} \times \mathbb{C}\right)$.

Case 3. $H_{1}^{\mathbb{}} \times \mathbb{R}$. The algebra on $H_{1}^{\llbracket} \times \mathbb{R}$ is given by

$$
\begin{aligned}
d e^{i} & =0, \quad \text { for } i=1, \ldots 5 \\
d e^{6} & =e^{1} \wedge e^{2}-e^{3} \wedge e^{4} \\
d e^{7} & =e^{1} \wedge e^{3}+e^{2} \wedge e^{4} \\
d e^{8} & =e^{1} \wedge e^{4}-e^{2} \wedge e^{3}
\end{aligned}
$$

The Obata connection is given by $\nabla_{e_{1}} e_{1}=-3 e_{5} / 2$ and $\nabla_{e_{2}} e_{2}=\nabla_{e_{3}} e_{3}=e_{5} / 2$. 
We obtain

$$
\begin{aligned}
d f^{i} & =0, \quad \text { for } i=1, \ldots, 8,13, \\
d f^{9} & =\frac{1}{2}\left(3 f^{1} \wedge f^{5}+f^{2} \wedge f^{6}+f^{3} \wedge f^{7}+f^{4} \wedge f^{8}\right), \\
d f^{10} & =\frac{1}{2}\left(-f^{1} \wedge f^{6}+3 f^{2} \wedge f^{5}-f^{3} \wedge f^{8}+f^{4} \wedge f^{7}\right), \\
d f^{11} & =\frac{1}{2}\left(-f^{1} \wedge f^{7}+f^{2} \wedge f^{8}+3 f^{3} \wedge f^{5}-f^{4} \wedge f^{6}\right), \\
d f^{12} & =\frac{1}{2}\left(-f^{1} \wedge f^{8}-f^{2} \wedge f^{7}+f^{3} \wedge f^{6}+3 f^{4} \wedge f^{5}\right), \\
d f^{14} & =f^{5} \wedge f^{6}-f^{7} \wedge f^{8}, \\
d f^{15} & =f^{5} \wedge f^{7}+f^{6} \wedge f^{8}, \\
d f^{16} & =f^{5} \wedge f^{8}-f^{6} \wedge f^{7},
\end{aligned}
$$

and therefore $d v_{i}=0$, for $i=1, \ldots, 4$, and $\left(d v_{7}\right)^{2,0}=\left(d v_{8}\right)^{2,0}=0$. Also $\left(d v_{5}\right)^{2,0}=$ $\frac{1}{2} v_{1} \wedge v_{3}$ and $\left(d v_{6}\right)^{2,0}=-\frac{1}{2} v_{1} \wedge v_{4}+v_{2} \wedge v_{3}$.

Choose $\mu_{0}=-\frac{3}{2} \bar{q}_{3} q_{3}$ and

$$
\begin{aligned}
\mu_{1}+i \mu_{2} & =\frac{1}{2}\left(q_{1} q_{3}-q_{2} \bar{q}_{4}\right)-\bar{q}_{1} q_{3}, \\
\mu_{3}+i \mu_{4} & =\frac{1}{2}\left(q_{2} \bar{q}_{3}+\bar{q}_{1} q_{4}\right), \\
\mu_{5}+i \mu_{6} & =\frac{1}{2}\left(\bar{q}_{1}-q_{1}\right), \\
\mu_{7}+i \mu_{8} & =-q_{2} .
\end{aligned}
$$

Then the ansatz (2) satisfies the HKT equation for $T^{*}\left(H_{1}^{\llbracket} \times \mathbb{R}\right)$.

In all three cases $g$ restricts to $g^{0}$ on the zero section $G$ in $T^{*} G$. Hence we actually obtain a positive definite metric in a neighbourhood of the zero section.

ACKNOWLEDGEMENTS. Birte Feix acknowledges the support of a Marie-Curie Individual Fellowship held at the University of Southern Denmark and of the Gott Research Fellowship at Trinity Hall, Cambridge. She also thanks the Department of Mathematics and Computer Science at the University of Southern Denmark for hospitality during the final stages of this paper. Lise and Henrik Pedersen are grateful to Gonville \& Caius College and Trinity Hall for a wonderful stay at an important stage of the work. Both authors would like to thank Anna Fino for many helpful discussions.

\section{REFERENCES}

1. M. L. Barberis, Affine connections on homogeneous hypercomplex manifolds, J. Geom. Phys. 32 (1999), 1-13.

2. N. B. Boyom, Models for solvable symplectic Lie groups, Indiana Univ. Math. J. 42 149-168 (1993). (2000). 
4. I. G. Dotti and A. Fino, Hyperkähler torsion structures invariant by nilpotent Lie groups, math. DG 0112166 (2001). $33-46$.

5. B. Feix, Hyperkähler metrics on cotangent bundles, J. Reine Angew. Math. 532 (2001),

6. B. Feix, Hypercomplex manifolds and hyperholomorphic bundles, Math. Proc. Camb. Phil. Soc. 133 (2002), 443-457.

7. G. Grantcharov and Y. S. Poon, Geometry of hyper-Kähler connections with torsion, Commun. Math. Phys. 213 (2000), 19-37.

8. P. S. Howe and G. Papadopoulos, Twistor spaces for hyper-Kähler manifolds with torsion, Phys. Lett. B 379 (1996), 80-86.

9. J. Michelson and A. Strominger, Superconformal multi-black hole quantum mechanics, JHEP 09, 005 (1999).

10. S. Salamon, Complex structures on nilpotent Lie algebras, J. Pure Appl. Algebra 157 (2001), 311-333. 